\title{
Numerical Modeling of the Stream Dynamics for River Channels with Complex Spatial Configuration
}

\author{
V.A.Shlychkov * \\ Institute for Water and Environmental Problems of SB RAS (Novosibirsk Branch) \\ 630090 Novosibirsk, Morskoy pr., 2, Russia
}

\begin{abstract}
Mathematical modeling provides a particularly important tool for studying the stream runoff formation processes, and its role is enhanced in the case of a sparse, obsolete monitoring network characteristic of most regions of Siberia. When analyzing spatio-temporal regularities of the water and sediment runoff in river systems, serious problems are caused by lack of the basic hydrological model capable of handling real-time data of hydrological measurements.

Calculations of unsteady flows in stream channels draw heavily on one-dimensional numerical models which are relatively easy to use and yield reliable results. Numerical investigations into the hydraulic regime of natural streams involve specific difficulties caused by the presence of nonlinear frictional forces in a turbulent flow, a variability in the channel geometry, the braiding of flows, the presence of floodplain depressions, riffles, etc. For especially complex stretches of rivers, a onedimensional approximation no longer fits the reality sufficiently adequately, so that the planar flow structure must be taken into account. For this purpose Saint Vennant's plane system of equations was used as the basis in order to develop further the numerical model due to this author which is intended for calculating the flow field, flow rates, levels, and impurity concentrations in natural water bodies of an arbitrary configuration or in a part of them. Fundamental laws of fluid mechanics are used as the basis for the model.

Spatial modeling of flows in complex regions necessitates reliable, consistent methods providing acceptable accuracy. As far as hydrological problems are concerned, the control volume method that allows the use of curvilinear grids was found to be the most powerful tool for obtaining the initial finite-difference relations.

This paper offers a number of examples illustrating the capabilities of the planar model for streams which is intended to resolve real problems arising at the design, construction and operation
\end{abstract}

*E-mail: slav@ad-sbras.nsc.ru 
stages of engineering structures in river channels and floodplains.

Key words: numerical modelling, hydrodynamics, planar flows, complex channel systems AMS subject classification: 86A05

\section{Introduction}

Mathematical description of hydrodynamical, channel and ice processes in complex plane-geometry rivers is among the most complicated problems in continuum mechanics [1]. The complexity of numerical models is caused by a number of factors, such as:

- the nonlinearity of basic equations of hydrodynamics;

- the presence of free boundaries in the form of bank lines with a previously unknown spatial configuration;

- the turbulent nature of the movement of river flows;

- the need to use a large body of experimental data on channel and floodplain topography, and on the physical and mechanical properties of banks and bottom material, and;

- the development of a state-of-the-art software for field data processing and for presentation of numerical experimentation results.

The theory of one-dimensional transient flows [2] can be regarded as the most advanced to date. Traditionally, the numerical solution to practical problems in river hydraulics is based on using the Saint-Venant equations in which the longitudinal coordinate is directed along the channel. This approach is quite justified for scales of hundreds and thousands of kilometers characteristics of Siberia's rivers, and one-dimensional models provide acceptable accuracy. For particularly complex stretches of rivers, a one-dimensional approximation turns out to be of little use for the reality, so that it is necessary to take into account the planar flow structure. This is particularly true for morphologically complicated stretches of stream channels with flow partitioning, braided pattern, riffles, and floodplains as well as for stretches of rivers with man-made water-development works.

The development of the computer-assisted planimetric model includes the following principal stages:

- analysis of natural factors and hydraulic characteristics of the fluvial stretch. Selection of source material containing hydrometric and morphometric field data;

- mathematical setting of the problem. Development of methods of discretization and numerical solution to systems of equations;

- development of the digital terrain model (DTM) at points of a regular grid with prescribed spatial resolution;

- generation of the curvilinear finite-difference grid with consideration for the geometry of the solution domain and the requirements for spatial detailing ensuring the desired calculation accuracy;

- interpolation of DTM to curvilinear grid points, bathymetric analysis of the depth field, and updating of incorrect values of the elevation matrix; 
- generation of initial fields and boundary conditions for the numerical model;

- investigation of the model sensitivity and making adjustments to using actual data;

- calculations, analysis and interpretation of results;

- development of image georeferencing tools.

Numerical models are based on fundamental laws from fluid mechanics providing the means of obtaining reliable quantitative estimates. The formulation of the planimetric model uses the SaintVenant equations in a two-dimensional approximation which permit reproduction of the spatial structure of flows in water bodies and streams with an accuracy required for practical purposes. The calculated hydrodynamic parameters of the flow serve as a kinematic basis in models of sediment transport and channel deformation and of the transport and diffusion of multicomponent impurities as well as in the study of the processes of frazil ice drift and ice jamming at the period of ice run after break-up.

\section{Model and methods}

Let us introduce the Cartesian coordinates with the axes $x, y$ in the horizontal plane. The surface of the channel bed is specified by the equation $z=z_{b}(x, y)$, where $z_{b}$ is a function describing topography. The equations for planar streams will be written as [3]:

$$
\begin{gathered}
\frac{\partial h u}{\partial t}+\frac{\partial h u u}{\partial x}+\frac{\partial h u v}{\partial y}=-g h \frac{\partial(h+\delta)}{\partial x}-\frac{g}{C_{s}^{2}}|\vec{u}| u+\frac{\partial \tau_{x x}}{\partial x}+\frac{\partial \tau_{x y}}{\partial y}+F_{x}, \\
\frac{\partial h v}{\partial t}+\frac{\partial h u v}{\partial x}+\frac{\partial h v v}{\partial y}=-g h \frac{\partial(h+\delta)}{\partial y}-\frac{g}{C_{s}^{2}}|\vec{u}| v+\frac{\partial \tau_{y x}}{\partial x}+\frac{\partial \tau_{y y}}{\partial y}+F_{y}, \\
\frac{\partial h}{\partial t}+\frac{\partial u h}{\partial x}+\frac{\partial v h}{\partial y}=0
\end{gathered}
$$

where $t$ is time, $h$ is flow depth (Figure 1), $u, v$ are velocity components, $g$ is gravity acceleration, $C_{s}$ is Chezy discharge coefficient; $\vec{u}=(u, v), \tau_{x x}=h K D_{T}, \tau_{x y}=h K D_{T}=\tau_{y x}=h K D_{S}$, $\tau_{y y}=-h K D_{T}$ are turbulent friction stresses, $D_{T}=u_{x}-v_{y}, D_{s}=v_{x}+u_{y}$ are the components of plane deformation, and $F_{x}, F_{y}$ are surface wind stresses. Using the Manning formula we can write $C_{s}=h^{1 / 6} / n$, where $n$ is a bottom roughness coefficient.

The description of exchange processes that not resolved explicitly within the accepted grid resolution uses one of the versions of the Smagorinsky model designed for parameterization of subgrid scales in numerical models for a planar horizontal flow [4]. The coefficient of turbulent exchange $K$ is calculated from the relation

$$
K=\alpha \frac{\Delta s}{2} \sqrt{D_{T}^{2}+D_{S}^{2}}
$$

where $\Delta s$ is the area of an elementary cell, and $\alpha$ is the proportionality factor. The formulation of the boundary conditions is determined by morphological characteristics of the water body, and by 


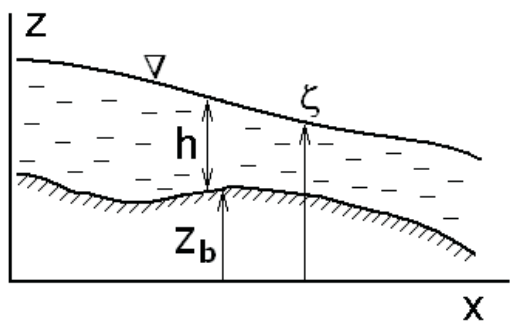

Figure 1: Longitudinal-transverse cross-section of a river channel.

the type of water exchange, and they are specified with consideration for the characteristics of the water stream. For describing the flows within a closed water body, it is necessary to specify on the side walls the conditions of no leakage and friction on the side walls. In the event that open (liquid) boundaries are present, the water flow rates $Q_{x}, Q_{y}$, must be known for the specified locations.

Basic algebraic relations were obtained by the finite-volume method. Curvilinear grids with the points positioned on the edges of an elementary spatial simulation box were used [5]. The system of equations (2.1) is projected into a nonrectangular elementary box with its sides similar in the direction to the curvilinear boundaries of the channel. Such an approach provides detailing of the transverse structure of the flow with a relatively small number of grid points located on longitudinal generatrices which roughly retrace the channel meanders. An integration over time was performed by the finite-differences method with the use of implicit algorithms. Spatial discretization of differential operators is based on the TVD schemes (Total Variation Diminishing) ensuring monotonicity of the solution through the use of the rearranging pattern and an appropriate selection of the approximation of derivatives in different segments of the numerical solution. The implicit portion of the TVD operators was algorithmized in accordance with the method described in [6].

\section{Results}

The planimetric model thus formulated, was tested against some stretches of rivers of a complex configuration. The problem of reconstructing the flow structure of the Lena river in the vicinity of Yakutsk was used as one of the case studies. With the flow rate $Q=5400 \mathrm{~m}^{3} / \mathrm{s}$ at the period of the lowest water level, the width of the main channel is $1-2 \mathrm{~km}$, and the depth reaches 12 meters in some places. Figure 2 shows the geometry of the channel in plan with elements of curvilinear grid and the distribution of depths. The water surface has a relatively complex configuration: the large Khalystakh island divides the stream into two arms: the navigable left arm, and the shallow right arm (the streamline is indicated by the arrows, and the existing navigation pass is shown by the solid line). When the water level rises, the island is partially inundated by a branch connecting both arms. In the bight in front of the island, the stream is undergoes a constraint because of the narrowing of the channel. Yakutsk is situated the left bank; its location is labeled by 1. Abreast of the front termination of the island, a cargo birth is located on the right riverside, which has the functions of the point of reloading (labeled by 2 in Figure 2). 


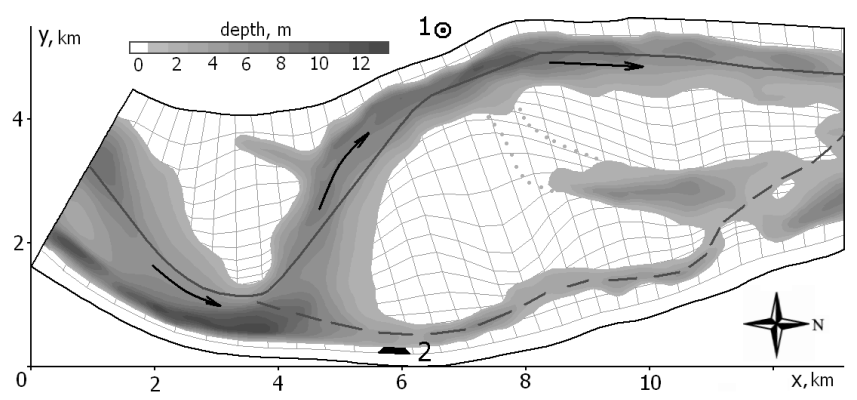

Figure 2: Planimetric configuration of channel and field of depths for Lena River stretch in the vicinity of Yakutsk city. 1 - Yakutsk city location, 2 - cargo terminal.

To optimize the operation of the transport junction, it is planned to relocate the navigable pass to the right arm (the dashed line in Figure 2). For this purpose it is envisaged to carry out bottom dredging and construct a system canals from the birth wall to the place of confluence of the arms in order to secure the adequate depth of $2.2 \mathrm{~m}$ required for pilotage at the period of the lowest water level. The reconstruction of the channel will alter the kinematic parameters of the stream, which in turn would cause a disturbance of the natural behavior of the channel processes, i.e. scouring of some parts of the bottom and silting of the others (and of drainage cuts in particular) due to planar migration of sediments.

The objective of a numerical modeling was to reconstruct the spatial structure of the kinematic parameters of the flow for the in-situ conditions and for the case of an altered channel morphology. In either case, it is necessary to investigate the channel for stability for the case of a high flow rate at the period of the lowest water level and for the case of an increase of the flow rate during floods. The channel-forming flow rate, i.e. the flow rate where the main sediment runoff occurs and channel deformations are most active, is $10400 \mathrm{~m}^{3} / \mathrm{s}$ for the Lena river stretch in the vicinity of Yakutsk.

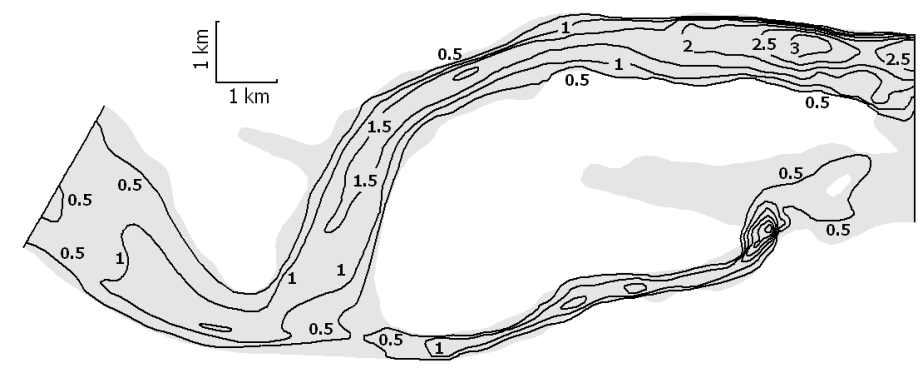

Figure 3: Calculated distribution of stream velocities in the channel.

The problem of studying the channel process was solved in two stages [7]. The $u, v, h$ fields corresponding to the specified flow rate were calculated first by integrating equations (2.1). The second stage involved studying the channel transformation on the basis of the calculated distribution of velocities and flow rates. The desired flow parameters under in-situ conditions were obtained by integrating over time the dynamical problem until a steady state was reached. The 
kinematic regime of the flow is characterized by the mean velocity of $1-1.5 \mathrm{~m} / \mathrm{s}$ I the main channel which in some places increases to $3 \mathrm{~m} / \mathrm{s}$ (Figure 3). The increase in the velocity is caused by the bottom rises in the left arm, and by the presence of submerged riffle in the right arm. In these places there arises a transverse skewness of the free surface. On the whole, the dynamical axis of the flow is found to be stable in the integration process, and the characteristics reach a steady state uniformly throughout the domain approaching a steady-state asymptote.

Since the springtime sediment-transporting flow is usually overloaded with sediments, there arises a hazard situation where alluvium can be deposited or the bottom of the artificial drainage cut is scoured, with the channel-forming flow rate $Q=10400 \mathrm{~m}^{3} / \mathrm{s}$. With a change in the hydraulic conditions, the self-regulation mechanisms of the "flow-channel" system are destroyed. In the case of insufficient flow velocities, siltation of the bottom is a potential hazard. Siltation is due to the transit transport of suspended sediment loads and is regulated by the transporting capacity of the flow. If the flow rate of sediment loads is larger than the transporting capacity, suspended sediments will accumulate in the channel; otherwise bottom scouring will occur. The balance of exchange processes implies the dynamical equilibrium of the sediment-transporting flow and of the bottom. To investigate the channel stability requires a calculation of the velocities of bottom deformations for the period of a spring flood. An analysis of the rate of change of the bottom profile is carried out in terms of $b_{t}=\frac{\partial z_{b}}{\partial t}$, reflecting the tendency of the load transportation vector [8].

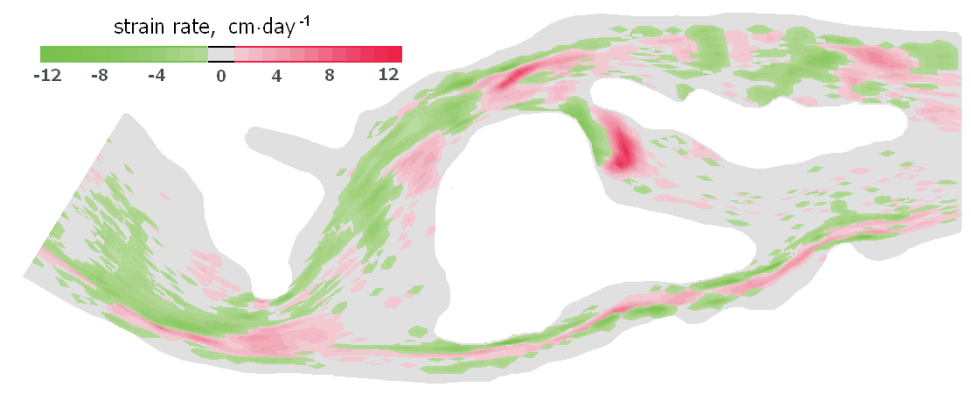

Figure 4: Field of bottom strain rates $b_{t}$ under conditions of dominant formative discharge. Areas of bottom erosion are indicated by green color, areas of bedload accumulation - by red color. Neutral areas are indicated by grey color.

Figure 4 illustrates the planimetric distribution of $b_{t}$ along the channel. Grey color corresponds to the equilibrium process with bottom elevations changing little, green color indicates areas with bottom erosion $\left(b_{t}<0\right)$, and red color shows areas with sedimentation $\left(b_{t}>0\right)$. It is evident from Figure 4 that the field of deformation rates has a low-contrast character, with the erosion phenomena predominating. The channel process is distributed uniformly in the river stretch distance, and only in the upper part of the branch connecting the two arms does there occur a local reshaping of the bottom in a shallow place.

The change in the bottom relief presented above characterizes the instantaneous initial values of the deformation rate when the bottom morphology remains unaltered. With the passage of time, the bottom profiles will also be changing over the course of channel deformations, and the flow will dynamically adjust itself to these changes. As a result, within a certain (relatively long) period 
of time, this will result in formation of other actual topography fields, fields of levels, and velocity fields; therefore, so that the problem needs to be solved anew on the basis of the new structural data. Since the straightforward solution to this problem in terms of the two-dimensional model is highly resource-intensive even for state-of-the-art computers, we do not discuss it in this paper. In general it can be stated that the anticipated reconfiguration of the right arm of the river to enable pilotage will result in a change of the character of channel deformation which, on the average, will present a siltation hazard along the length of the arm.

All water bodies of Siberia are covered in ice for a protracted period of time. Springtime ice movements are accompanied by fractures and fragmentation of the ice cover along the river channel into separate ice fields and floes which are transported by the flow downstream. In the case of a high density of the floating ice massif, the ice field can stop moving in the narrowing stretches of the river due to blockage of the ice drift core in the restrained stream. This results in an ice jam, with its thickness increasing with time because of incorporation of new masses of floating floes. Ice jams lead to a rapid rise of the water level in the river, and to disastrous floods causing huge material damage. Thus, an extremely large ice jam and flood in the vicinity of Lensk (Yakutia) almost totally destroyed the urban agglomeration in 2002, and the city is being rebuilt to date. The stretch of the Lena river near Yakutsk is also characterized by conditions for regular formation of springtime ice jams.

There are as yet no universal methods for studying jam-hazardous river stretches. A dynamicalstochastic hybrid model of spring ice drift is presented in what follows, in which the flow parameters along the channel are reconstructed in terms of the deterministic numerical model, while and the floating ice floes are treated as different-scale elements of a stochastic ensemble. Also, the dynamics of a separate ice element is calculated individually under some hypotheses of pairwise interaction with nearby ice floes, including with those forming part of the ice jam region [8].

A model planar flow for the Lena river, calculated by neglecting the ice effects, was specified as hydrodynamical field when modeling the ice run (Figure 3).

The coordinates of the initial set of ice ensemble elements were specified using the random number generator with a uniform distribution. The size of the floes was specified using the normal distribution law with the value of the diameter averaging $150 \mathrm{~m}$, and with the variance of $50 \mathrm{~m}$. The density of the ice run was taken to be 0.4 . These figures were maintained in the generation process of new elements at the entrance river range in lieu of those traveling further downstream. The water surface area within computational domain is $26.7 \mathrm{~km}^{2}$, and the total area covered with ice floes is $10 \mathrm{~km}^{2}$. The total number of the ensemble elements in the domain does not exceed 1500 .

Numerical experiments showed that with a low density of the ice run and in the absence of large elements, the floating ice travels in transit along the channel and leaves the computational stretch through the exit range. Only in a relatively narrow right arm, at the passage of large floes there is a high likelihood that a local ice jam is produced, which will have no influence the passage of the ice along the main (left) stream.

With an increase in the sampling variance and in the element size, the main channel can be clogged by one or several floes because of their being jammed by the banks. Observations indicate that the main factor that is responsible for the delay in movement of the fragmented ice is the 
constraint of the flow due to the narrowing of the channel. In this case there emerges an ice bridge spanning the channel which serves as the site of origin (core) of the ice jam. This bridge hinders a free floating of the ice and causes the floes to accumulate behind the core thereby leading to an increase in the extent of the ice jam field over time.

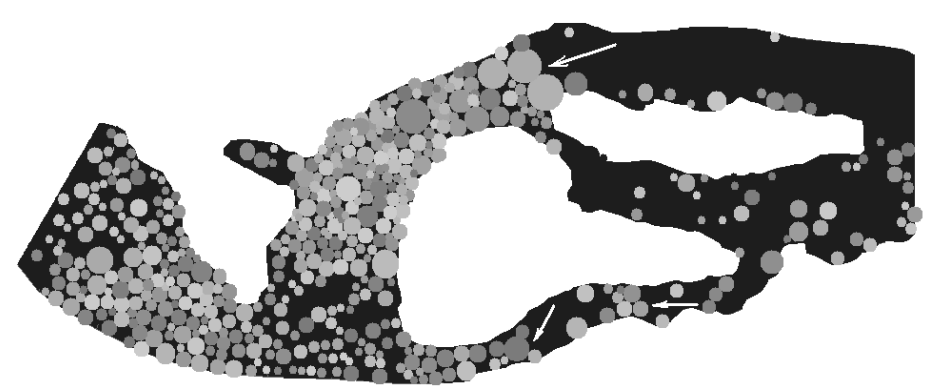

Figure 5: Configuration of ice ensemble during the channel clogging in the Lena River. Arrows indicate jam cores.

The jam configuration of the ensemble at the time of forming of the ice obstacle is shown in Figure 5. The channel of the left arm is spanned by a group of large floes nearby the connecting branch due to the effects of thrust and friction on the banks (shown by the arrows). It should be noted that the delay of movement of the ice here is the result of the naturally occurring process of interaction between the ensemble and the channel geometry, i.e. it mimics a natural phenomenon in terms of a model representations. A number of small jams are also produced in the left arm.

As the mass of ice accumulates in the rear part of the ice jam, there is an increase in longitudinal stresses within the ice cover, the closely packed ice fragments are acted upon by excess pressure, so that some of the floes are crushed and squeezed out from their horizontal. The ice jam experiences a hummocking, accompanied by an increase in the thickness of the ice field and its downward displacement by gravity. Subsidence of the ice cover leads to a narrowing of its cross-section, while an increase in roughness of the lower surface gives rise to an increase in total hydraulic resistance. These effects are reproduced on the basis of theoretical concepts of the mechanism of floe collision. A decrease in clear section decreases the channel capacity, i.e. is responsible for changes in the conditions of movement of the water flow, as a result of which there occurs its deceleration and a level rise at the end of the ice jam. Calculations showed that a zone of hydrostatic head was produced within 1.5 hour after the channel blockage; the water level in this zone exceeded more than $1 \mathrm{~m}$ that in the ice-free flow.

The dynamical-stochastic model presented above is the first (or one of the first) model of this kind for the description of the ice run. Its advantage is the abandonment of the conventional concept of the continuum, i.e. the field of fragmented ice is represented not as a continuous substance but as an ensemble of discrete elements, which, undoubtedly, is more realistic and permits more reliable reconstruction of the ice drift parameters. An obvious practical focus of this research involves identifying jam-hazardous river stretches and assessing the likelihood of channel blockage according to the statistical characteristics of the ice run. Routine prediction results can be obtained by the statistical test method through a multivariate run of model ensembles with different sets of 
characteristics corresponding to the scatter of parameters of a real ice situation.

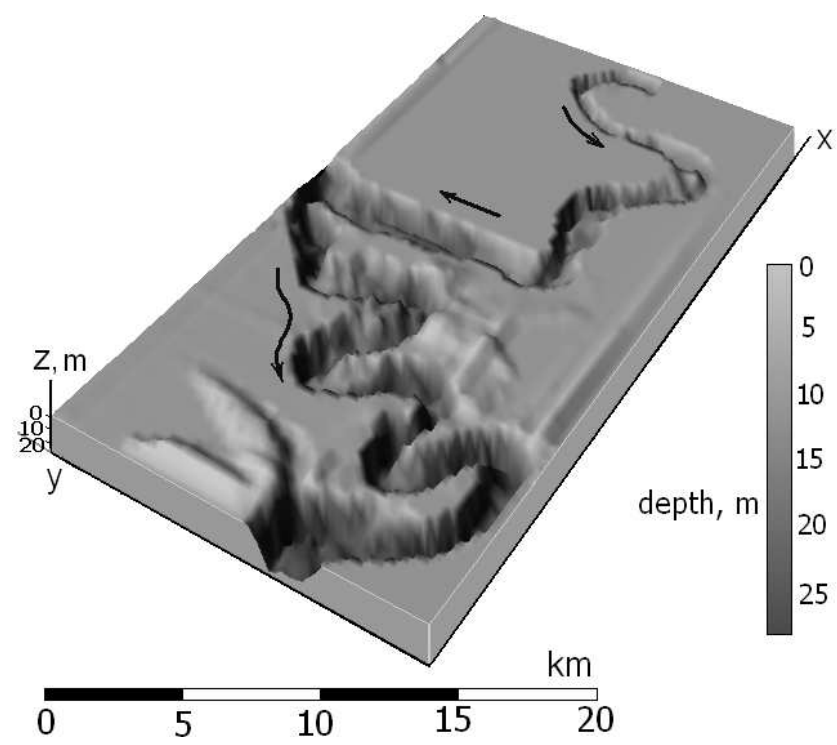

Figure 6: Three-dimensional image of the Chusovaya River channel morphometry.

Let us consider one further example illustrating a calculation of channel flows of a complex geometry. Figure 6 shows the spatial structure of the Chusovaya river channel (left tributary of the Kama river) flowing in the foothills of the Western Ural Mountains (the flow direction is indicated by the arrows). The river stretch under consideration is under the influence of the hydrostatic head of the Kama reservoir; the channel is heavily meandering and is distinguished by a complex spatial structure. The length of river stretch is about $80 \mathrm{~km}$ along the geometrical axis.

Reconstructing the flow pattern in the Chusovaya river is of interest for the following reason. The river mouth hosts an intake facility to supply water to the nearby city of Perm with the population size in excess of 800 thousand. In the upper reaches of the river there are springs with high salinity, so that the entry of their waters into the intake heads is undesirable. To work out the hydraulic engineering measures in order to prevent salt-affected water intake requires a detailed knowledge of the flow structure along the entire length of the channel, and it is the planimetric model that serves these purposes. Another aspect of the problem is related to the development of a simplified model of the river through an appropriate reduction of the planimetric problem a one-dimensional model for the purpose of constructing the numerical model for the Kama reservoir with its length totaling $340 \mathrm{~km}$ ). In such a model, the relatively small Chusovaya river will turn out to be one in a series of hydrological fragments; it will suffice to describe it by a simplified method without too much sacrifice of quality of the description. To accomplish this, the two-dimensional structure of the flow is studied to generate, on its basis, a hydraulically equivalent one-dimensional model which is a component of the general model of the reservoir.

Figure 7(a) presents the calculated flow pattern in terms of the stream function for the specified water flow rate $Q=1000 \mathrm{~m}^{3} / \mathrm{s}$. The streamlines describe the trajectories of particles and, at the same time, form stream tubes in which the value of the water flow rate is constant (in Figure 7(a), the 


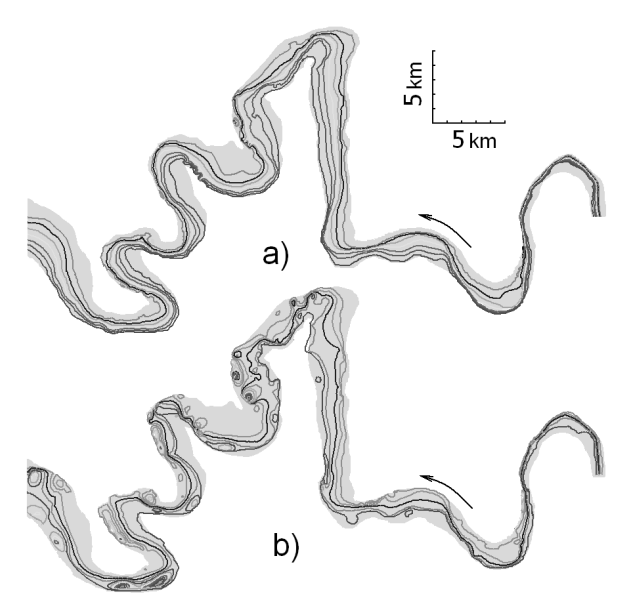

Figure 7: Isolines of stream function in Chusovaya River at $Q=1000$ (a) and $Q=2000 \mathrm{~m}^{3} / \mathrm{sec}$ (b).

stream tubes release jets with identical flow rates, $125 \mathrm{~m}^{3} / \mathrm{s}$ ). With an increase of the flow rate, the flow velocity increases, and the dynamical effects give rise to eddy circulations at the periphery of the stream (in Figure 7(b), the streamlines were obtained for $Q=2000 \mathrm{~m}^{3} / \mathrm{s}$ ). Eddies develop in the river meanders and in places of significant topographic non-uniformity of the channel and banks; their influence manifests itself as a considerable distortion of the streamlines relative to a calm flow. Large eddy areas produce reverse flows, velocity pulsations are increased in them, and a turbulent exchange is well developed. To formulate a one-dimensional model, which is identical to a one-dimensional model as regards the quality of the description, is a nontrivial endeavor, given the conditions of intense generation of eddies, and the complex flow structure.

\section{Conclusion}

Mathematical modeling is a powerful (and, in many applications, the only) tool for forecasting and assessing the eventual consequences of the impact of natural and technogenic factors on the functioning and development of complex hydrogeographical systems. Nowadays the creation of an adequate mathematical model for a water body under study is practically a "must" in developed countries. Powerful techniques have been developed to date for solving hydraulic problems, based on planimetric equations for complex-configuration channel flows. The model, developed by this author and presented in this paper, includes the basic hydrodynamic unit, and a number of submodels, such as the ice thermics unit, and impurity transport unit. The latter is designed to solve ecological problems of pollutants transport in rivers as well as problems of suspended sediment transport and channel deformation. This study has covered the main aspects related to the use of the model series in the exploration of hydrological and geographical problems, such as the spatial differentiation of the types of hydrological regimes in river channels, and animated mapping of channel and ice processes for risk assessment of adverse natural phenomena. 


\section{References}

[1] O. Vasiliev. Mathematical modelling of hydraulic and hydrologic processes in water reservoirs and streams (Review of the RAS Siberian Branch publications). Vodnye resursy, 26 (1999), No. 5. 600-611 (Russian).

[2] A. Atavin, O. Vasiliev, A. Voevodin, S. Shugrin. Numerical methods of solution to onedimensional problems of hydrodynamics. Vodnye resursy, 20 (1993), No. 4. 38-47 (Russian).

[3] J. Stoker. Water waves: the mathematical theory with applications. Moscow. Foreign Literature Publ, 1959 (Russian).

[4] J. Smagorinsky. General circulation experiments with the primitive equations: 1 . the basic experiment. Mon. Weather Rev., 91 (1963), No 2, 99-164.

[5] B. Sanders. High resolution and non-oscillatory solution of the St. Venant equations in nonrectangular and non-prismatic channels. J. Hydrauloc Res., 39 (2001), No. 3, 236-244.

[6] A. Harten. On a class of high resolution total-variation-stable finite-difference schemes. SIAM Journal of Numerical analysis, 21 (1984), No. 1, 1-23.

[7] V. Degtyarjov, Y. Dolzhenko, V. Shlychkov. Hydrotechnical construction of navigable waterways of Yakutsk traffic centre. Novosibirsk, Agros, 2007 (Russian).

[8] V. Shlychkov. Calculation of channel currents and transport of sediments on the basis of plain model for Novosibirsk reservoir. Proc. 10 International Symposium on River Sedimentation, Moscow, MGU, 3 (2007), 284-291. 